\title{
Bases for the correct determination of resonance integrals of reactions leading to isomeric states: application to some reactions induced on zinc
}

\author{
M.S. Segovia ${ }^{1}$, M.C. Fornaciari Iljadica ${ }^{1, a}$, M.A. Arribére ${ }^{2}$, and I.M. Cohen ${ }^{3}$ \\ ${ }^{1}$ National Atomic Energy Commission, Ezeiza Atomic Centre, Presbítero González y Aragón No 15, B1802AYA Ezeiza, Argentina \\ 2 National Atomic Energy Commission, Bariloche Atomic Centre, Avenida Bustillo, Km 9.7, 8400 Bariloche, Argentina \\ 3 National Technological University, Buenos Aires Regional Faculty, Department of Chemical Engineering, \\ Avenida Medrano 951, C1179AAQ Buenos Aires, Argentina
}

\begin{abstract}
The problems related with the departures from the ideal behaviour of the epithermal component of the neutron spectrum are discussed, in connection with the measurement of resonance integrals. The characteristics of the methods for the measurement of the $\alpha$ parameter are commented and the bases of a new method for the determination of mean effective resonance energy, developed at the authors' laboratory, are briefly described. On these grounds, the application to the correct measurement of the resonance integrals of ${ }^{68} \mathrm{Zn}(\mathrm{n}, \gamma)^{69 \mathrm{~m}} \mathrm{Zn},{ }^{70} \mathrm{Zn}(\mathrm{n}, \gamma)^{71} \mathrm{Zn}$ and ${ }^{70} \mathrm{Zn}(\mathrm{n}, \gamma)^{71 \mathrm{~m}} \mathrm{Zn}$ is presented.
\end{abstract}

\section{Introduction}

One of the problems that the users of tabulated nuclear data on reactor neutron-induced reactions find is the existence of discrepant values. Some of the reasons of such discrepancies [1], which constitute a serious drawback in connection with the choice of the "best" or most appropriate values, are:

- use of non-consistent input data sets;

- absence of updated input values, either related with the studied reactions or with those eventually used as standards;

- "optimistic" evaluation of the uncertainties associated to the determinations;

- incomplete information about the specific convention adopted to describe the neutron flux;

- lack of adequate characterization of the neutron spectrum employed.

Regarding capture reactions produced by epithermal neutrons, the latter is possibly the most relevant factor, since the actual flux at the different positions frequently shows departures from the ideal behaviour. Thus, the values of the resonance integrals determined in a particular spectrum can not be considered as universal data, unless a correction is performed. As it will be explained in the next section, both the $\alpha$ parameter, associated with the irradiation position, and the mean effective resonance energy, characteristic of the precursor, must be well known in order to accomplish this calculation.

This work deals with the determination of resonance integrals of some reactions leading to isomeric states, based on a precise measurement of the $\alpha$ parameter and also of the mean effective resonance energies, by a new experimental method developed at the authors' laboratory.

${ }^{a}$ Presenting author, e-mail: iljadica@cae.cnea.gov.ar

\section{The actual epithermal spectrum and its implications}

On the basis of theoretical considerations, it is possible to describe the slowing-down of neutrons in an ideal, infinite and non-absorbing medium, as a function of the inverse of the energy. However, the actual epithermal spectrum of a reactor shows a $\left(\mathrm{E}^{1+\alpha}\right)^{-1}$ dependence [2], where $\alpha$ is a parameter, usually within 0.1 and -0.1 , which corrects the departures from the ideal behaviour at the irradiation position.

Therefore, any experimental measurement performed at a nuclear reactor refers to $\mathrm{I}_{0}(\alpha)$, a resonance integral that differs from that defined for an ideal spectrum. The expression of this magnitude is:

$$
I_{0}(\alpha)=\left[\frac{I_{0}-2\left(\frac{E_{0}}{E_{C d}}\right)^{1 / 2} \sigma_{0}}{\left(\bar{E}_{r}\right)^{\alpha}}+\frac{2\left(\frac{E_{0}}{E_{C d}}\right)^{1 / 2} \sigma_{0}}{(2 \alpha+1) E_{C d}^{\alpha}}\right] 1 e V^{\alpha}
$$

where $\mathrm{I}_{0}$ : resonance integral in an ideal spectrum; $\sigma_{0}$ : crosssection at the reference energy $\mathrm{E}_{0}(0.025 \mathrm{eV}$ at $293.2 \mathrm{~K}) ; \mathrm{E}_{\mathrm{Cd}}$ : cadmium cut-off energy (usually taken as $0.55 \mathrm{eV}$ ); $\bar{E}_{r}$ : mean effective resonance energy.

The most relevant aspect of the consideration of the departures from the ideal behaviour of the epithermal spectrum is that the nuclear constants obtained in a reactor are not directly applicable to other reactors, nor those measured in a particular irradiation position with respect to other ones of the same reactor. Thus, many of published data on resonance integrals and $\mathrm{Q}_{0}$ values (resonance integral to cross-section ratios) could be at least questionable.

It can be seen, from equation (1), that the conversion of the experimentally determined $\mathrm{I}_{0}(\alpha)$ values to $\mathrm{I}_{0}$ data requires the previous knowledge of the $\alpha$ parameter and the mean effective resonance energies, in connection with the irradiation position and the precursors of the reactions, respectively.

The measurement of $\alpha$ presents some difficulties: some of the methods [3-5] require either irradiation of at least three bare flux monitors or of two monitors irradiated both in the 
whole neutron spectrum and in the epithermal component, with the use of cadmium screenings. A most recent method, developed at the authors' laboratory [6], is based on a single irradiation under cadmium cover of two selected monitors, with combined values of $\mathrm{Q}_{0}(0)$ and $\bar{E}_{r}$ that allow a simplified calculation. Nevertheless, the main problem is associated with the currently used values for mean effective resonance energies, calculated from the resonance parameters of the target [7], because they should not be used when the products are isomeric states.

\section{Experimental}

Two irradiation positions of the RA-6 reactor (Bariloche Atomic Centre) were employed, one of them at the reactor core (F5) and the other, predominantly thermal, surrounded by the graphite reflector (I6). The typical values of the thermal flux in these positions are $7 \times 10^{12} \mathrm{n} . \mathrm{cm}^{-2} \mathrm{~s}^{-1}$ and $8 \times 10^{11}$ n.cm ${ }^{-2} \mathrm{~s}^{-1}$, respectively, whereas the subcadmium to epicadmium flux ratios (f) are 35 and 100.

The preparation of the samples, all irradiated inside small cadmium boxes, depended on the physical state of each target. Powders were irradiated in small sealed polyethylene bags and metallic samples as wires, foils or sheets. Very small deposits on filter papers, from specially prepared solutions, were also used [8].

Irradiation, decay and measurement times were selected according to the characteristics of each of the radionuclides produced. The measurements were carried out by high resolution gamma spectrometry.

\subsection{Determination of the $\alpha$ parameter}

The method described in ref. [6] was selected for the determination of the $\alpha$ parameter. As it was already mentioned, the foundation of this method is the simultaneous irradiation of two targets under cadmium cover and the use of an approximate formula for the $\mathrm{Q}_{0}(\alpha)$ expression. Gold and zirconium samples, respectively as deposits on filter paper and small metallic foils, were used. The results of replicate measurements, at F5 and I6 positions, were -0.0239 and 0.0266 , both with uncertainties of $10 \%$.

\subsection{Determination of the mean effective resonance energies}

In addition to the method described in ref. [7], other experimental method has been published [9], which requires the measurement of the $\alpha$ parameter in several positions (seven, in the original publication). Because of this requisite, its accomplishment is somewhat cumbersome and its use has been restricted. Taking into account these aspects, a new method was developed [8,9]. In what follows, a brief description of this method will be provided.

The counting rate of a sample irradiated in a reactor flux can be expressed by:

$$
C=N \phi_{e p i} \sigma_{0} i \varepsilon S D M\left[f+Q_{0}(\alpha)\right]
$$

where $\mathrm{N}$ : number of atoms of the precursor; $\phi_{\text {epi }}$ : epicadmium flux per unit ln E; i: emission probability of the radiation measured with $\varepsilon$ efficiency; S, D, and M: temporal factors related with the irradiation, decay and measurement times.

Using the $\mathrm{k}_{0}$ formalism [10] and reordering the equation (2):

$$
\frac{C}{k_{0} m S D M \varepsilon}=\phi_{e p i}\left[f+Q_{0}(\alpha)\right] \frac{N_{A v} \theta_{A u} i_{A u} \sigma_{0, A u}}{A_{r, A u}}
$$

$\mathrm{m}$ is the mass of the element, $\mathrm{N}_{\mathrm{Av}}$ the Avogadro's number, $\mathrm{A}_{\mathrm{r}}$ the relative atomic mass and $\theta$ the isotopic abundance. The left member of this equation will be denoted by $\mathrm{W}$.

$$
\frac{N_{A v} \theta_{A u} i_{A u} \sigma_{0, A u}}{A_{r, A u}}
$$

is a constant expressed through Avogrado's number and well known gold constants. For a particular irradiation position, the product between this constant and $\phi_{\text {epi }}$ is also a constant, which will be expressed with $\mathrm{K}$. Therefore, the equation results as follows:

$$
W=K\left[f+Q_{0}(\alpha)\right]
$$

The different values of $\mathrm{Q}_{0}(\alpha)$ as a function of $\mathrm{W}$ are described by a straight line with slope $\mathrm{K}$ and ordinate $\mathrm{Kf}$.

$\mathrm{W}$ is proportional to the activity and also to $\mathrm{Q}_{0}(\alpha)$. Its value is specific for each nuclide irradiated in a particular position. The relative weight of $\mathrm{Q}_{0}(\alpha)$ increases for lesser $\mathrm{f}$ values; therefore, irradiations under cadmium cover $(f \approx 0)$ are advisable.

From a series of $\mathrm{W}$ values and the $\mathrm{Q}_{0}(\alpha)$ constants calculated for nuclides selected as standards, calibration curves can be drawn, so that it is possible to find the value of an unknown $\mathrm{Q}_{0}(\alpha)$. If two irradiation positions, very well differentiated trough their $\alpha$ values, are used, two different $\mathrm{Q}_{0}(\alpha)$ are obtained. Thus, the following system can be formulated:

$$
\begin{aligned}
& Q_{0}\left(\alpha_{1}\right)=\frac{W_{1}-K_{1} f_{1}}{K_{1}}=\frac{Q_{0}(0)-0.429}{\overline{E_{r}^{\alpha_{1}}}}+\frac{0.429}{\left(1+2 \alpha_{1}\right) 0.55^{\alpha_{1}}} \\
& Q_{0}\left(\alpha_{2}\right)=\frac{W_{2}-K_{2} f_{2}}{K_{2}}=\frac{Q_{0}(0)-0.429}{\overline{E_{r}^{\alpha_{2}}}}+\frac{0.429}{\left(1+2 \alpha_{2}\right) 0.55^{\alpha_{2}}} .
\end{aligned}
$$

As it is easily observed, this equation system allows the calculation of both the mean effective resonance and the $\mathrm{Q}_{0}(0)$ values, from which the resonance integrals can be derived. It is worthwhile mentioning that this system gives the bases for the experimental determination of all these constants and is also applicable to isomeric states.

\subsection{Selection of the nuclides for standardization and validation}

The primordial condition for the selection of the nuclides destined to the calibration curves was that none of them led to isomeric states by capture reactions. In addition, special attention was paid to the published values of $\mathrm{Q}_{0}(0)$ factors and mean effective resonance energies. Nuclides with excessively high effective resonance energies were avoided. The range 
of $\mathrm{Q}_{0}(0)$ values of the calibration curves was wide enough, in order to be in conditions to solve an ample variety of unknowns.

The following nuclides and their respective reactions were used for calibration: ${ }^{55} \mathrm{Mn}(\mathrm{n}, \gamma){ }^{56} \mathrm{Mn} ;{ }^{65} \mathrm{Cu}(\mathrm{n}, \gamma)$ ${ }^{66} \mathrm{Cu} ;{ }^{74} \mathrm{Ge}(\mathrm{n}, \gamma){ }^{75} \mathrm{Ge} ;{ }^{75} \mathrm{As}(\mathrm{n}, \gamma){ }^{76} \mathrm{As} ;{ }^{74} \mathrm{Se}(\mathrm{n}, \gamma){ }^{75} \mathrm{Se} ;{ }^{98} \mathrm{Mo}$ $(\mathrm{n}, \gamma){ }^{99} \mathrm{Mo}$; and ${ }^{186} \mathrm{~W}(\mathrm{n}, \gamma){ }^{187} \mathrm{~W}$.

The methodology was validated through the investigation of the nuclear constants connected with the capture reaction on ${ }^{152} \mathrm{Sm}$, which leads to the formation of an only product, ${ }^{153} \mathrm{Sm}$. The half-life of this nuclide allows its measurement at relatively long periods; in addition, the energy of the gamma emission is adequate for a good detection. The results of the determination of the resonance integral and the mean effective resonance energy were entirely satisfactory [9].

\section{Application to some reactions on zinc, leading to isomeric states}

The criteria applied in the definition of unknowns were related to the following aspects: lack of data for the epithermal activation of some nuclides or, alternatively, existence of values that could be considered as dubious or questionable, when the influence of the $\alpha$ parameter was not included as part of the determination, or when the determination involved isomeric pairs without discrimination of the reactions, in connection with the mean effective resonance energies.

Some reactions induced on zinc isotopes present challenges of this kind: whereas some values of the epithermal activation parameters have been published for the ${ }^{68} \mathrm{Zn}(\mathrm{n}, \gamma)^{69 \mathrm{~m}} \mathrm{Zn}$ reaction, there are practically no data related with the reactions leading to the ${ }^{71} \mathrm{Zn} /{ }^{71 \mathrm{~m}} \mathrm{Zn}$ isomeric pair. Since ${ }^{69 \mathrm{~m}} \mathrm{Zn}$ is an isomeric state, the objections formulated with respect to the datum for the mean effective resonance energy are sustained.

The relevant data of the activation constants and the characteristics of the products were extracted from ref. [11-13].

\subsection{Results and discussion}

The value of $\mathrm{Q}_{0}(0)$ found for the ${ }^{68} \mathrm{Zn}(\mathrm{n}, \gamma)^{69 \mathrm{~m}} \mathrm{Zn}$ reaction is $3.592 \pm 0.081$. From this datum, and using the value of the cross-section published in ref. [13], $(0.072 \pm 0.004) b$, the final value for the resonance integral is $(0.259 \pm 0.016) b$.

The literature value for this reaction (ref. [14]) is $(0.24$ \pm 0.03 ) b. No evidence exists about the consideration of the $\alpha$ parameter in the generation of this value. If this parameter was included, the mean effective resonance energy used would have been that calculated from the resonance parameters of the target, ${ }^{68} \mathrm{Zn}$, which should not be applied to the isomeric state. Any of these factors could explain the differences observed between the published value and the obtained in the present work. Besides its less uncertainty, and because of what discussed in the preceding paragraphs, the latter can be considered as more reliable.

The final values of $\mathrm{Q}_{0}(0)$ for the ${ }^{70} \mathrm{Zn}(\mathrm{n}, \gamma)^{71} \mathrm{Zn}$ and ${ }^{70} \mathrm{Zn}(\mathrm{n}, \gamma)^{71 \mathrm{~m}} \mathrm{Zn}$ reactions are, respectively, $1.51 \pm 0.28$ and
$21.85 \pm 0.87$. Using again the cross-section data contained in ref. [13], the values for the resonance integrals are $(0.125$ $\pm 0.025) \mathrm{b}$ and $(0.190 \mathrm{~b} \pm 0.013) \mathrm{b}$. Only working values, determined at the authors' laboratory, have been published for these reactions [15]. They are $(0.1350 \pm 0.0093) b$ and $(0.157 \pm 0.029) b$. Both were measured under the assumption that the corrections derived from the $\alpha$ parameter were not too significant, because the value of this parameter was not high at the position used. In spite of the similitude between these previous values and the present ones, these latter should be adopted as completely reliable, since the characteristics of the actual spectrum were taken into account in the determination.

In conclusion, the methodology presented in this work is rigorous and directly applicable to the correct determination of resonance integrals, with special emphasis in the isomeric states. The obtained results have been adequately validated and could represent a valuable contribution to the literature on this subject.

\section{References}

1. M.A. Arribére, I.M. Cohen, A.J. Kestelman, S. Ribeiro Guevara, M. Arrondo, M.C. Fornaciari Iljadica, Proyecciones 3(1), 37 (2005).

2. T.B. Ryves, Metrologia 5, 119 (1969).

3. F. De Corte, L. Moens, A. Simonits, A. De Wispelaere, J. Hoste, J. Radional. Chem. 52, 295 (1979).

4. F. De Corte, L. Moens, K. Sordo-El Hammami, A. Simonits, J. Hoste, J. Radioanal. Chem. 52, 305 (1979).

5. I.M. Cohen, J.C. Furnari, E.H. Montoya, M.A. Arribere, A.J. Kestelman, P. Mendoza, B. Torres, in Proceedings of the Second International $k_{0}$ Users Workshop, Ljubljana, Slovenia, 30 Sept. -3 Oct. (1996).

6. I.M. Cohen, M. Arrondo, M.A. Arribére, M.C. Fornaciari Iljadica, Nucl. Sci. Eng. 154, 110 (2006).

7. L. Moens, F. De Corte, A. Simonits, A. De Wispelaere, J. Hoste, J. Radioanal. Chem. 52, 379 (1979).

8. M.S. Segovia, M.Sc. thesis, National Technological University, 2006.

9. M.S. Segovia, M.C. Fornaciari Iljadica, I.M. Cohen, Proyecciones 5(1) (2007) (in press).

10. A. Simonits, F. De Corte, J. Hoste, J. Radional. Chem. 24, 31 (1975).

11. K.J. Tuli, Nuclear Wallet Cards, 6th edn., National Nuclear Data Center, Brookhaven National Laboratory, 2000.

12. M.-M. Bé, V. Chisté, C. Dulieu, E. Browne, V. Chechev, N. Kuzmenco, R. Helmer, A. Nichols, E. Schönfeld, R. Dersch, Monographie BIPM-5, Table of Radionuclides, Vol. 1 - A = 1 to 150. Bureau International des Poids et Mesures, 2004.

13. S.F. Mughabghab, M. Divadeenam, N.E. Holden., Neutron Resonance Parameters and Thermal Cross Sections (Academic Press, New York, 1981).

14. E.M. Gryntakis, D.E. Cullen, G. Mundy, in Handbook on $\mathrm{Nu}$ clear Activation Data, IAEA Technical Reports Series 273, (1987), p. 199.

15. I.M. Cohen, S. Riveiro Guevara, M.A. Arribére, M.C. Fornaciari Iljadica, A.J. Kestelman, R.A. Ohaco, M.S. Segovia, A.N. Yunes, Radiochim. Acta 93, 543 (2005). 